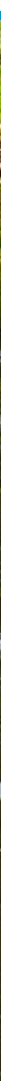

Nachsorge ist Vorsorge

\title{
Start der Kommunikations-Initiative zur Nachsorge für Kinder und Jugendliche nach Krebs
}

Köln: Für Kinder und Jugendliche nach einer Krebserkrankung gibt es jetzt erstmals einen individuellen Nachsorge-Kalender mit begleitender Informationsbroschüre. Die Broschüre richtet sich an Kinder und Jugendliche nach der Behandlung eines Knochentumors (Osteosarkom/Ewing-Sarkom) und erscheint im Rahmen einer Broschürenreihe, die folgende fünf Einzelbroschüren umfasst: .Weichteilsarkome, Leukämie, Hirntumoren, Lymphome, Embryonale Tumoren.

\section{Hilfen für strukturierte Nachsorge}

Die Broschürenreihe als erster Teil der Kommunikations-Initiative „Nachsorge ist Vorsorge" soll bei Kindern und Jugendlichen, die eine Krebserkrankung überlebt haben, das Bewusstsein dafür stärken, dass nach Abschluss der onkologischen Behandlung ein erhöhtes Risiko für Spätfolgen der Erkrankung und ihrer Behandlung besteht und daher eine strukturierte Nachsorge erforderlich ist. Kalender und Broschüre informieren Kinder und Jugendliche sowie deren Eltern über die Wichtigkeit einer kontinuierlichen Krebsnachsorge. Die Informationsmaterialien werden durch den behandelnden Arzt im Rahmen der Abschlussuntersuchung abgegeben. Im weiteren Verlauf können Ärzte, die die Nachsorge-Untersuchung durch- führen, die Befunde direkt in den Nachsorge-Kalender eintragen. Dadurch lässt sich stets auf einen Blick erkennen, wann bei welchem Arzt welche Nachsorgeuntersuchungen durchgeführt wurden und wann eine erneute Untersuchung ansteht. So wird die lückenlose Beurteilung der gesundheitlichen Situation und ihrer Behandlung möglich.

\section{Aus Nachsorge wird Vorsorge}

Dank der erheblichen Fortschritte in der Therapie von Krebserkrankungen im Kindes- und Jugendalter wird die Gruppe der Überlebenden immer größer. Diese Erfolge verlangen aber oft eine recht aggressive Behandlung. Nachsorge ermöglicht es, Gesundheitsstörungen als Folge der Krebstherapie und eventuelle Rezidive oder Metastasen frühzeitig zu entdecken. Je früher, desto besser lassen sie sich behandeln. Krebsnachsorge ist somit zugleich Krankheitsvorsorge. Die Broschüren-Serie soll Hilfestellung und strukturierendes Element zur kontinuierlichen Nachsorge sein.“

Kontakt: Prof. Dr. med. Thorsten Langer, Late Effects Surveillance System (LESS), E-Mail: thorsten.langer@uk-erlangen.de 